\title{
A novel mice model of acute flares in osteoarthritis elicited by intra-articular injection of cultured mast cells
}

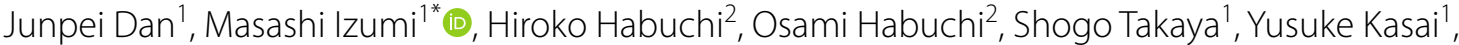
Ryuzo Hayashi ${ }^{3}$, Koji Aso ${ }^{1}$, Takahiro Ushida ${ }^{2}$ and Masahiko Ikeuchi ${ }^{1}$

\begin{abstract}
Purpose: Mast cells are multifunctional in osteoarthritis $(\mathrm{OA})$, and infiltration of activated mast cells likely contributes to disease severity and progression. However, the detailed mechanisms of action are unclear. The purpose of this study was to elucidate the role of mast cell infiltration in OA at histological level using a new mice model and to investigate pharmacological inhibitory effects of existing mast cell stabilizers in this model.

Methods: Mice were injected intra-articularly with monosodium iodoacetate (MIA $0.5 \mathrm{mg}$ ) or PBS on day 0 , and PBS, with or without mast cells (MC: $1 \times 10^{6}$ cells) on day 14 . They were divided into four groups: OA flare (MIA + MC), $\mathrm{OA}(\mathrm{MIA}+\mathrm{PBS}), \mathrm{MC}$ non-OA (PBS + MC), and PBS non-OA (PBS + PBS). In OA flare, the MC stabilizer drug (tranilast: $400 \mathrm{mg} / \mathrm{kg} /$ day) or PBS was administered intraperitoneally from days 15 to 21.

Results: Histologically, modified Mankin score of the OA flare was significantly higher than that of OA (7.0 [1.8] vs. $3.3[1.3], P<0.05)$, and a larger number of mast cells was observed in OA flare than in OA (34.5 [6.3]/mm² vs. 27.2 [2.3]/ $\left.\mathrm{mm}^{2}, P<0.05\right)$ on day 22. OA flare also showed acute exacerbation of pain and increased gene expression of proinflammatory cytokines and aggrecanase compared with OA. Administration of tranilast to OA flare-up provoked significant improvements in term of histological changes, pain, and gene expression at day 22.
\end{abstract}

Conclusion: Our novel model possibly mimics OA flare conditions, which may open a new strategy of disease-modifying treatment for $\mathrm{OA}$, focused on controlling the multiple functions of mast cells.

Keywords: Mast cell, Osteoarthritis, Pain, Inflammation, Flare

\section{Background}

Osteoarthritis $(\mathrm{OA})$ is the most common form of arthritis in the elderly and has long been considered a non-inflammatory condition characterized by progressive degeneration of articular cartilage [9]. However, a growing body of evidence has shown that local inflammation, that is, synovitis, plays an essential role in the pathophysiology of

\footnotetext{
${ }^{*}$ Correspondence: izumim@kochi-u.ac.jp

${ }^{1}$ Department of Orthopedic Surgery, Kochi Medical School, Kochi

University, 185-1 Oko-cho, Nankoku, Kochi Pref, Japan

Full list of author information is available at the end of the article
}

$\mathrm{OA}$ as it is a key predictor of joint failure and a determinant of pain [22].

Infiltration of immune cells into synovial tissue is an important phenomenon in the development and progression of OA. Although the underlying cellular and molecular mechanisms such as rheumatoid arthritis have not been fully elucidated, the most frequent types of immune cells found in OA joints are macrophages, $\mathrm{T}$ cells, and mast cells $(\mathrm{MC})[16,17]$. There is limited research in the role of MCs in the pathophysiology of OA as compared to that of macrophages and $\mathrm{T}$ cells. Previous reports from the 1990s, however, showed that the number of MCs increased in the synovium and synovial fluid, which 
was associated with the degree of inflammation in OA patients $[4,10,35]$. Recently, an immunohistochemistry study demonstrated that the number of activated MCs in the synovium was positively correlated with the severity of synovitis and radiological disease progression of knee OA [8]. These reports strongly suggest that synovial MC infiltration is associated with a variety of inflammatory changes, which play a key role in the pathophysiology of OA.

Pain is a major symptom of OA, and acute aggravation of pain between chronic stable states is frequently seen in clinical practice. This is known as 'pain flare' or 'flareup' [25]. Flares have been described as inflammatory in nature and are often prolonged for a while [25]. The underlying mechanisms of this condition, however, have not yet been fully described. Although a number of painful rodent OA models have been developed, there is inadequate basic research focusing on histological changes in the acute flare. Regarding treatment, coping strategies against the OA flare condition have not been established clinically [28]; hence, symptom-modifying drugs are often used continuously. However, adverse side effects are always problematic, especially in elderly patients [30].

In this study, we hypothesized that acute flares would develop partly because of activated MCs in the OA joint and the results of this study can help to identify a new disease-modifying approach focused on controlling the multiple functions of mast cells.

The objectives of this study were therefore:

1) To elucidate the role of mast cell infiltration in OA at histological level using a new mouse model mimicking the OA flare condition.

2) To investigate the pharmacological inhibitory effect of existing mast cell stabilizers in this model.

\section{Methods \\ Experiment 1: Development of OA flare model Animals}

Male C57BL/6 mice aged 7 weeks (18-20 g, Japan SLC, Hamamatsu, Japan) were housed under standard conditions. All experiments were carried out after 1 week of acclimatization to the environment. The experimental protocol was approved by the Institutional Review Board of our University for Animal Research (M-00078).

\section{Preparation of MC}

Bone marrow cells were isolated from the femurs of 8-week-old male C57BL/6 mice and cultured in RPMI 1640 medium containing $10 \%$ foetal bovine serum and $50 \%$ conditioned medium of WEHI-3 cells in plastic culture dishes [32]. The culture was continued for 5 weeks with weekly changes in the medium. Cell viability was determined by trypan blue staining. The concentration of the MC solution was then adjusted to $1 \times 10^{6}$ cells / $20 \mu \mathrm{l}$ PBS [11].

\section{Induction of $O A$ and intra-articular injection of $M C$}

OA was induced through a single intra-articular injection of monoiodoacetate (MIA), which is a well-established model associated with cartilage degeneration, joint destruction, and persistent pain behavior [31, 34, 46]. On day 0 , the mice were anaesthetized by isoflurane inhalation. After sterilising their knee with $70 \%$ ethanol, MIA (0.5 mg) dissolved in $10 \mu \mathrm{l}$ saline was injected into the right knee through the patellar tendon using a $30 \mathrm{G}$ Hamilton micro syringe. PBS was used as a control. On day 14 , the MC solutions $\left(1 \times 10^{6}\right.$ cells / $20 \mu \mathrm{l}$ PBS $)$ were injected into the ipsilateral knee using the same procedure as above. The concentration of the MC solution was determined according to a previous intradermal injection of local MC injection intra-dermally [11]. PBS was injected as a control instead of $\mathrm{MC}$ solution. For subsequent analyses, mice were divided into four groups as follows according to the injected solutions on day 0 and day 14: OA flare (MIA + MC), OA (MIA + PBS), MC non-OA $(\mathrm{PBS}+\mathrm{MC})$, and PBS non-OA (PBS + PBS) (Fig. 1). First, two groups (MIA + MC and MIA + PBS) were created to investigate the role of $\mathrm{MC}$ infiltration in the $\mathrm{OA}$ joint. As no reports were available for $\mathrm{MC}$ injection into a normal joint, the MC non-OA (PBS + MC) group developed secondarily. The PBS non-OA (PBS + PBS) group was used as control.

\section{Histopathological analysis}

Mice were euthanized on day 22, and their knees were extracted and fixed in $4 \%$ paraformaldehyde for one day, decalcified in $13 \%$ formic acid solution for 2 weeks, and then embedded in paraffin. The specimens were sagittally sectioned at $5 \mu \mathrm{m}$ thickness and stained with toluidine blue. Histological sections were observed using a Nikon ECLIPSE 80i microscope (Nikon, Tokyo, Japan). Histopathologic classification of the severity of OA was graded using a modified Mankin score ranging from 0 to 13 points ( 0 as best, 13 as worst) [47]. In addition, MCs were identified as cells with round or elliptical morphology and numerous cytoplasmic metachromatic granules [36] at the meniscosynovial junction. The number of MCs in the meniscosynovial junction was counted at $400 \times$ magnification. The area of the meniscosynovial junction was then measured using image analysis software (Image J) [37] and the number of MCs was normalised by the area (number/ $\mathrm{mm}^{2}$ ). 


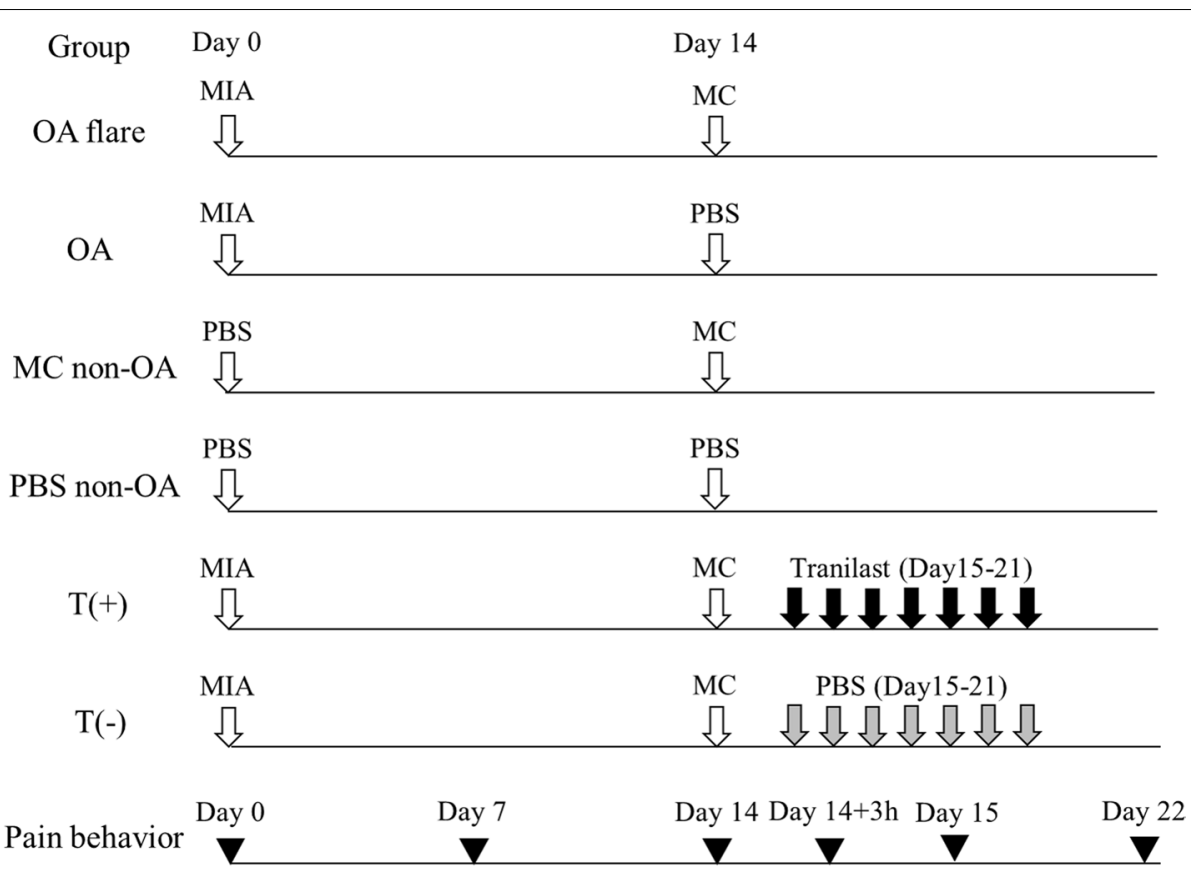

Fig. 1 Experimental design. Mice were divided into the following 4 groups according to injected solutions at day 0 and 14; OA flare (MIA + MC), $\mathrm{OA}(\mathrm{MIA}+\mathrm{PBS}), \mathrm{MC}$ non-OA (PBS + MC), and PBS non-OA (PBS + PBS) (experiment 1). Furthermore, in OA flare, the mice were divided into 2 groups depending on the tranilast injection $(T(+), T(-))$ (experiment 2). Pain behavior was measured at specific timing of days $0,7,14,14+3 \mathrm{~h}(3 \mathrm{~h}$ after injection), 15, and 22. Mice were euthanized at day 22; subsequently, histological analyses and RT-PCR were conducted

\section{Immunofluorescence staining}

Immunofluorescence staining was performed to confirm the distribution of injected (exogenous) MC over time. Green fluorescent protein (GFP) expressing MCs were obtained from C57BL/6-Tg (CAG-EGFP) mice provided by Japan SLC (Hamamatsu, Japan) cultured for 5 weeks, and they were injected into the OA knees (MIA injected 14 days ago) using the same protocol as above. Mice were euthanized at 1, 3, and 7 days after injection of GFPexpressing MCs, and paraffin-embedded sections of the knee were obtained at each point in time. The presence of MCs, including both exogenous and endogenous cells, was investigated using mast cell protease-6 (MCP-6) immunofluorescence staining. The knees were extracted and fixed in $4 \%$ paraformaldehyde for one day, decalcified with 0.5 M EDTA for 2 weeks, and then embedded in paraffin. Following deparaffinization in xylene and washing with graded alcohol, activation with $0.1 \%$ trypsin was performed at $37{ }^{\circ} \mathrm{C}$ for $30 \mathrm{~min}$. After blocking with $5 \%$ normal goat serum for GFP and 5\% normal donkey serum for MCP-6, the slides were incubated at room temperature $\left(20-25^{\circ} \mathrm{C}\right)$ for an hour. Primary antibodies against rabbit GFP (1:500, Novus Biologicals, CO, USA) and goat MCP-6 (1:500, Santa Cruz Biotechnology, TX, USA) were applied to the sections and incubated overnight at $4^{\circ} \mathrm{C}$. The next day, sections were incubated with the secondary antibody for $2 \mathrm{~h}$. Goat anti-rabbit IgG Alexa Fluor 546 (1:400, Thermo Fisher Scientific, MA, USA) and donkey anti-goat IgG Alexa Fluor 488 (1:400, Thermo Fisher Scientific, MA, USA) were used as the corresponding secondary antibodies. Before, between, and after each incubation step, the sections were washed three times for $5 \mathrm{~min}$ PBS. Finally, all sections were mounted with Vectashield (Vector, Burlingame, CA). Sections were viewed with an FV-1000D laser confocal microscope (Olympus, Tokyo, Japan) and GFP/MCP6 immuno-reactive cells were observed.

\section{Pain behavior test}

Mice were individually placed in a transparent container at room temperature, and their spontaneous activities were continuously recorded using a digital camera (Nikon, Tokyo, Japan) for $10 \mathrm{~min}$. Two observers independently watched the recordings and provided a semiquantitative evaluation of pain behavior using Stance score [12] as follows: $0=$ No visible impairment of gait or stance, the foot firmly placed flat on the surface with normal spread of toes. $1=$ Moderate impairment of stance, the foot is placed on the ground with toes tightly contracted. $2=$ Severe impairment of gait and stance; foot either entirely elevated from the ground or only the lateral part of the foot lightly touching the ground and toes 
tightly pulled together. As the 'stance' varied frequently during the observation period, the highest score maintained for at least $10 \mathrm{~s}$ was therefore assigned as the final score. Intermediate scores $(0.5$ and 1.5$)$ were used for animals that displayed a behavior in between the definitions described above. Scoring was performed on days 0 , $7,14,14+3 \mathrm{~h}$ (3 $\mathrm{h}$ after injection), 15, and 22 (Fig. 1). The average score from the two observers was used for data analyses. Ipsilateral paw hyperalgesia was assessed simultaneously. After $30 \mathrm{~min}$ of acclimatisation, paw withdrawal threshold was evaluated using von Frey filaments (North Coast Medical Inc., Morgan Hill, CA, USA) 1.65, 2.36, 2.44, 2.83, 3.22, 3.61, 3.84, 4.08, 4.17, and 4,31 (corresponding to $0.008,0.02,0.04,0.07,0.16,0.4,0.6,1.0$, 1.4 , and $2.0 \mathrm{~g}$, respectively).

\section{Real time-polymerase chain reaction (RT-PCR) analysis}

Total RNA was isolated from the right side of the infrapatellar fat pad on day 22, and mRNA levels of IL-1 $\beta$, TNF- $\alpha$, IL-6, ADAMTS- 4 , and NGF was measured by the quantitative RT-PCR intercalated method. cDNA was synthesised using the ReverTra Ace ${ }^{\circledR}$ qPCR RT Kit (Toyobo, Osaka, Japan). Reaction mixtures for RT-PCR were composed of $2 \mu \mathrm{l} \mathrm{cDNA}, 1.6 \mu \mathrm{M}$ specific primer pair, $10 \mu \mathrm{l}$ TB Green Premix Ex Taq II (Takara, Kyoto, Japan), $0.4 \mu \mathrm{l}$ ROX reference dye, and $6 \mu \mathrm{l}$ nuclease free water in a final volume of $20 \mu \mathrm{l}$. Quantitative PCR was performed using a real-time PCR system (StepOnePlus; Thermo Fisher Scientific, MA, USA) to detect the relative mRNA expression levels. The PCR cycle protocol was as follows: the holding stage performed one cycle at $95{ }^{\circ} \mathrm{C}$ for $30 \mathrm{~s}$, the cycling stage was repeated 40 times at $95{ }^{\circ} \mathrm{C}$ for $15 \mathrm{~s}$, and at $60{ }^{\circ} \mathrm{C}$ for $60 \mathrm{~s}$. mRNA expression was normalised to the levels of HPRT mRNA.

\section{Experiment 2: Treatment of OA flare model with tranilast}

To investigate the possibility of pharmacological suppression of MC-injection-induced acute flare, tranilast, an established MC stabilizer drug, was systemically administered to OA flare mice. This is according to a previous report using a collagen-induced arthritis model mimicking RA [14]. Tranilast has been used therapeutically for many years as an anti-allergic drug against bronchial asthma, allergic rhinitis, atopic dermatitis, and hypertrophic scars [9], and has been approved by the National Insurance System in our country. In the OA flare group, $1 \mathrm{ml}$ of tranilast $(400 \mathrm{mg} / \mathrm{kg} /$ day $)$ or PBS was injected intra-peritoneally with a $27 \mathrm{G}$ syringe once a day from day 15 to 21 . According to previous studies, tranilast was administered orally [40] or through an intraperitoneal injection [14], yet no study has been conducted using a local injection. In this study, we selected intraperitoneal injections to avoid differences in tranilast intake by oral administration. The mice were divided into two groups depending on the tranilast injection: the $\mathrm{T}(+)$ and $\mathrm{T}(-)$ groups. To evaluate the pharmacological effects of tranilast, histology (modified Mankin score and number of MCs), pain behavior (stance score), and RT-PCR (IL-1 $\beta$, TNF- $\alpha$, IL-6, ADAMTS-4, and NGF) were examined in an identical manner to the first experiment. An overview of the experimental protocol is presented in Fig. 1.

\section{Statistical analysis}

The primary outcome of this study was histological changes in the OA flare model compared with the OA model in Experiment 1, and the pharmacological inhibitory effects of tranilast against this altered histology in Experiment 2. Pain behavior and joint inflammation were set as secondary outcomes in both experiments. Our sample size was determined by referring to a previous study that examined the modified Mankin score in a mouse MIA OA model. In the study, it was found that a $20 \%$ decrease in this histological score after a specific treatment was statistically significant [1]. Calculations were performed according to this difference with $90 \%$ statistical power and $\alpha$ level of 0.05 , and it was determined that at least 10 mice were needed for OA flare and OA group in Experiment 1 and, $\mathrm{T}(+)$ and $\mathrm{T}(-)$ group in Experiment 2. Finally, a sample size of 13 mice were chosen to allow for possible dropouts. Kruskal-Wallis test followed by Mann-Whitney U test with Bonferroni correction was used to compare pain behavior, modified Mankin score, and MC number among the four groups (OA flare, OA, MC non-OA, and PBS non-OA). The Mann-Whitney $U$ test was used to compare pain behavior, modified Mankin score, and MC number with or without tranilast administration to the OA flare mice ( $\mathrm{T}$ $(+)$ vs. T (-) groups). To evaluate mRNA gene expression levels between the groups (OA flare vs. OA group, and $\mathrm{T}$ $(+)$ vs. T (-) group), the Mann-Whitney U test was also used. Statistical significance was set at $p<0.05$. All statistical analyses were performed using SPSS software (ver. 26.0; SPSS, Chicago, IL, USA).

\section{Results \\ Experiment 1}

A sufficient amount of viable MC was successfully obtained after five weeks of culture. Histologically, the modified Mankin score (median [interquartile range]) was 7.0 [1.8] in the OA flare group, which was significantly higher than $3.3[1.3]$ in the OA group $(P<0.05$; Fig. 2). The number of MCs (median [interquartile range]) also showed a significant difference among the groups (OA flare $34.5[6.3] / \mathrm{mm}^{2}$ vs. OA 27.2 [2.3] / $\mathrm{mm}^{2}, P<0.05$; Fig. 2). Immunofluorescence staining (A) 1 day, (B) 3 days, and (C) 7 days after MC injection 


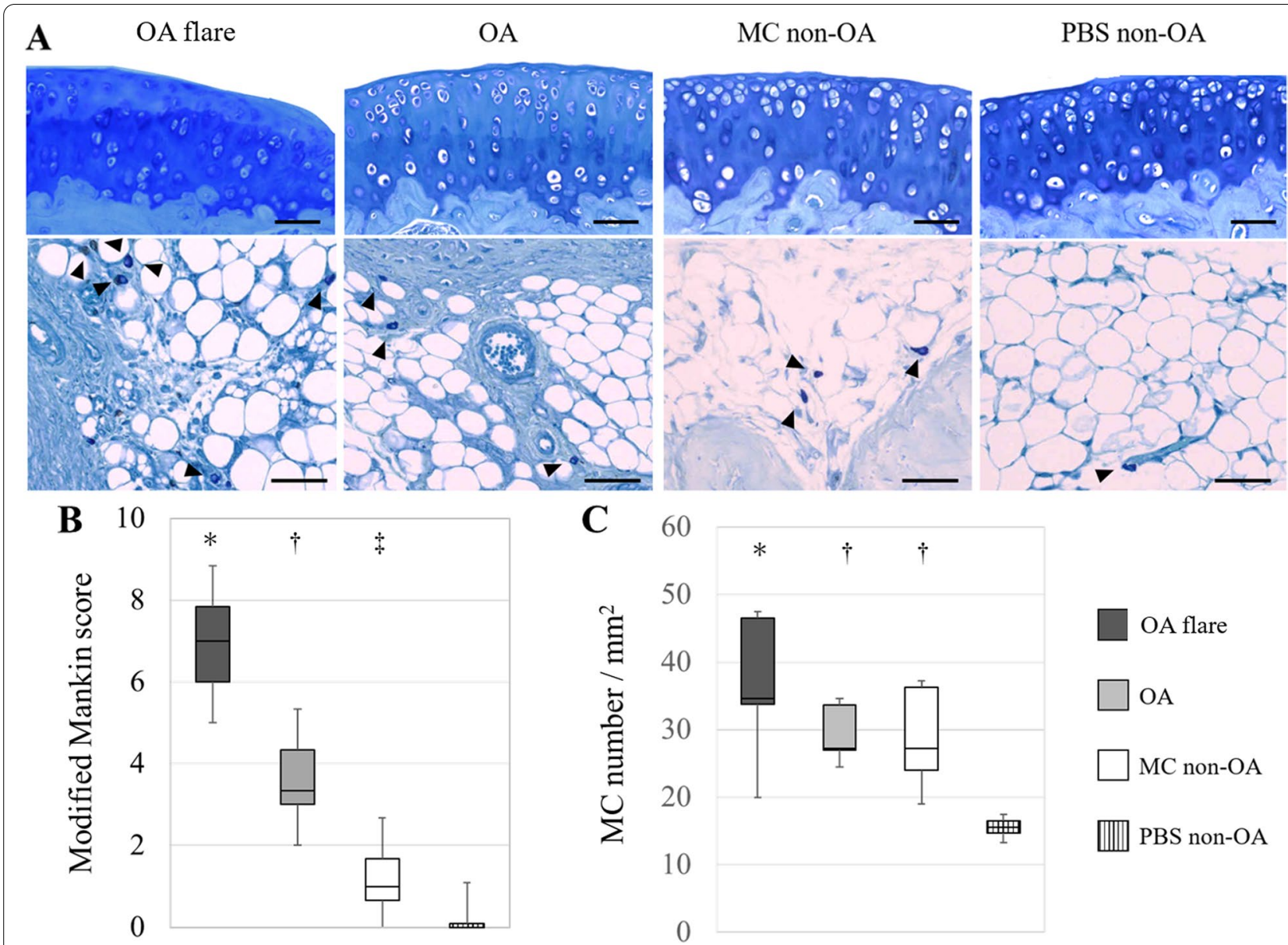

Fig. 2 A Histopathological changes of $O A$ and MC infiltration (toluidine blue staining). Arrowhead indicates MC. B Modified Mankin score (Median [interquartile range]). OA flare $(n=13), O A(n=13), M C$ non-OA $(n=16)$, and PBS non-OA $(n=4) . *$ : $P<0.05$ compared with OA, MC non-OA, and PBS non-OA; + : $P<0.05$ compared with MC non-OA and PBS non-OA; $\neq: P<0.05$ compared with PBS non-OA. $C$ Number of MC located in the synovium around the meniscosynovial junction. (Median [interquartile range]). ${ }^{*}: P<0.05$ compared with OA, MC non-OA, and PBS non-OA; + : $P<0.05$ compared with PBS non-OA. (Black bar: $50 \mu \mathrm{m})$

showed that MC labelled with GFP and MCP-6 was observed in (A) and (B), which demonstrated that exogenous MC existed at that time. MCP-6 positive cells were still present in (C), but GFP-positive cells were not observed, suggesting that endogenous MC accounted for the majority 7 days after MC injection (Fig. 3). MIA injection induced significant pain and paw hyperalgesia compared to PBS injection $(P<0.05$; Fig. 4$)$. After the second injection at day 14, Stance score (median (range)) was $1.0(0.5-2.0)$ in the OA flare group which was significantly higher than $0.5(0-1.0)$ in the OA group $(P<0.05$; Fig. 4). However, this difference was not detected in von Frey test. The MC non-OA group showed no pain and paw hyperalgesia, which was comparable to the PBS non-OA group (Fig. 4). The mRNA expression levels of IL-1 $\beta$, TNF- $\alpha$, IL-6, ADAMTS-4, and NGF were 1.5-2.8 times higher in the OA flare group than in the OA group $(P<0.05 ;$ Fig. 5$)$.

\section{Experiment 2}

Histologically, the modified Mankin score was 4.8 [1.2] in the $\mathrm{T}(+)$ group, which was significantly lower than $6.3[1.5]$ in the $\mathrm{T}(-)$ group $(P<0.05$; Fig. 6$)$. The number of MCs also showed a significant difference among the groups $\left(\mathrm{T}(+) 17.3[4.2] / \mathrm{mm}^{2}\right.$ vs. $\mathrm{T}(-) 31.8[11]$ $/ \mathrm{mm}^{2}, P<0.05$; Fig. 6). Regarding the pain behavior, both $\mathrm{T}(+)$ and $\mathrm{T}(-)$ groups showed similar pain on day 15 with the Stance score of $1.0(0.5-1.5)$ in $\mathrm{T}(+)$ group and $1.0(0.5-2.0)$ in $\mathrm{T}(-)$ group $(P=0.54)$. On day 22 , the stance score was reversed to $0.5(0-0.5)$ in the $\mathrm{T}(+)$ group following repeated tranilast injections; however, this change was not observed in the $\mathrm{T}(-)$ group $(P<0.05)$. The mRNA expression levels of IL-1 $\beta$, TNF- $\alpha$, IL- 6 , ADAMTS-4 were $0.35-0.75$ times lower in the $\mathrm{T}(+)$ group than in the $\mathrm{T}(-)$ group $(P<0.05$; Fig. 6). 


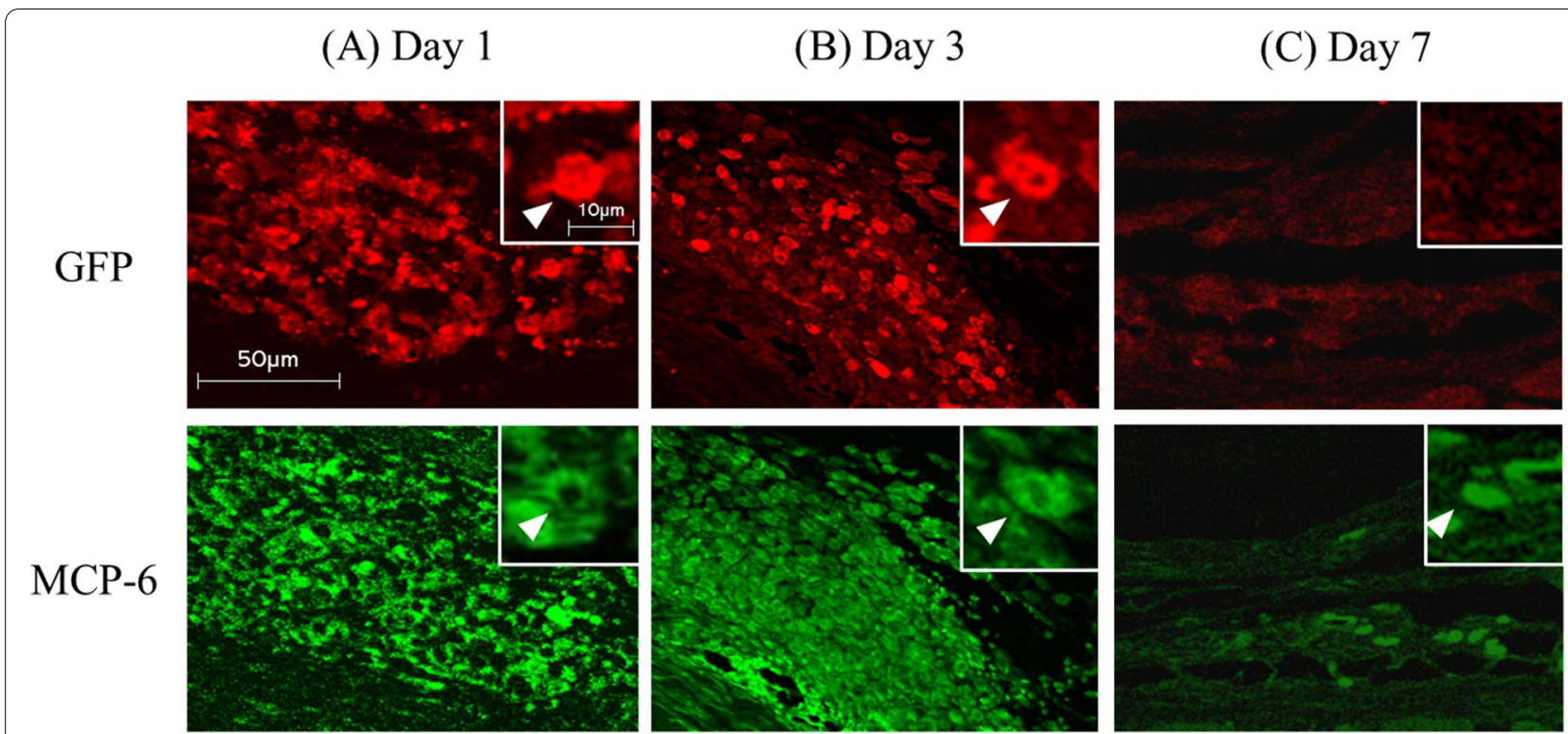

Fig. 3 Representative photos presenting distributions of injected MC in the knee joint over time. Immunofluorescence staining (A) 1 day, (B) 3 days, and (C) 7 days after the MC injection. Cells labelled with GFP and MCP-6 were observed in (A) and (B), which demonstrated that exogenous MC existed at that time. MCP-6 positive cells still existed in (C), but GFP positive cells were not observed, suggesting that endogenous MC accounted for the majority 7 days after the MC injection. Magnification was identical in all 6 panels

A

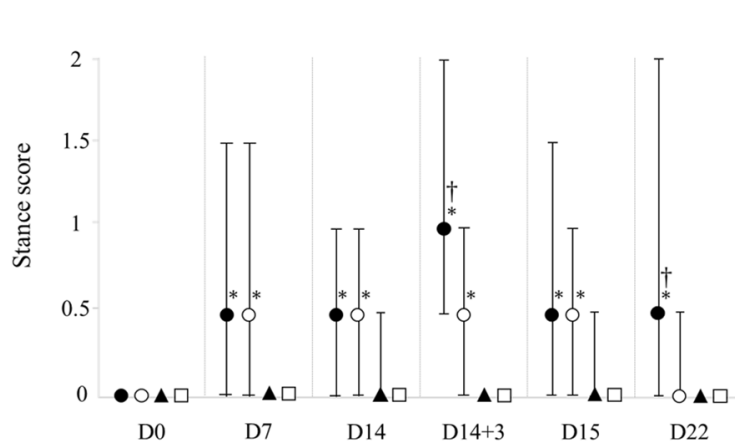

B

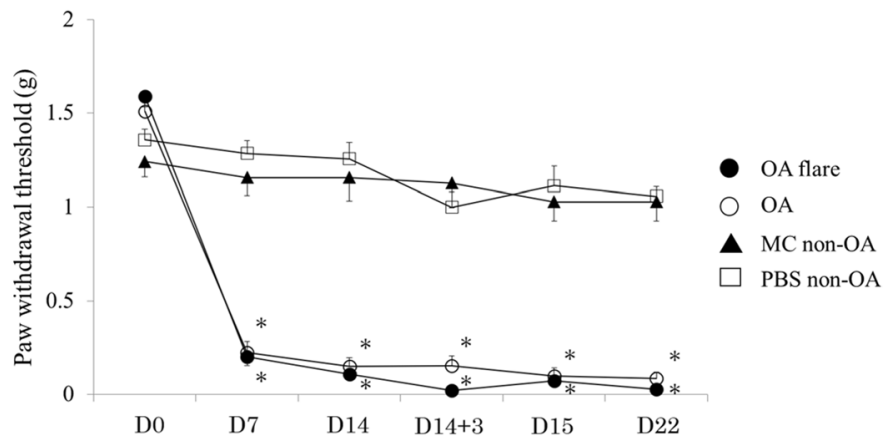

Fig. 4 A Median (range) of Stance score in each group at day 0 before the first injection (D0), day 7 (D7), day 14 before the second injection (D14), $3 \mathrm{~h}$ after the second injection (D14 + 3), day 15 (D15), and day 22 (D22). B Mean (SEM) of ipsilateral paw withdrawal threshold in each group at D0, $D 7, D 14, D 14+3, D 15$, and D22. OA flare (filled circle, $n=31$ ), OA (open circle, $n=30$ ), MC non-OA (triangle, $n=12$ ), and PBS non-OA (square, $n=9$ ), respectively. *: $P<0.05$ compared with $\mathrm{PBS}$ non-OA; + : $P<0.05$ compared with $\mathrm{OA}$

\section{Discussion}

This study focuses on the role of $\mathrm{MC}$ infiltration in the OA joint to clarify some of the underlying mechanisms of acute OA flare conditions. In clinical practice, symptom-modifying drugs are generally used for this condition; however, they often have limited effects on disease progression. In addition, adverse events associated with the continuous use of this drug are not rare, especially in elderly patients. The results of this study can help to identify a new disease-modifying approach focused on controlling multiple function of MC. Our novel OA flare mice model showed articular cartilage degeneration together with $\mathrm{MC}$ infiltration at histological level, acute exacerbation of pain behavior, and increased gene expression of proinflammatory cytokines and aggrecanase in the knee joint compared to the conventional MIA OA mice model. Furthermore, systemic administration of tranilast in the OA flare model significantly suppressed histological deterioration, pain behavior, and gene expression 


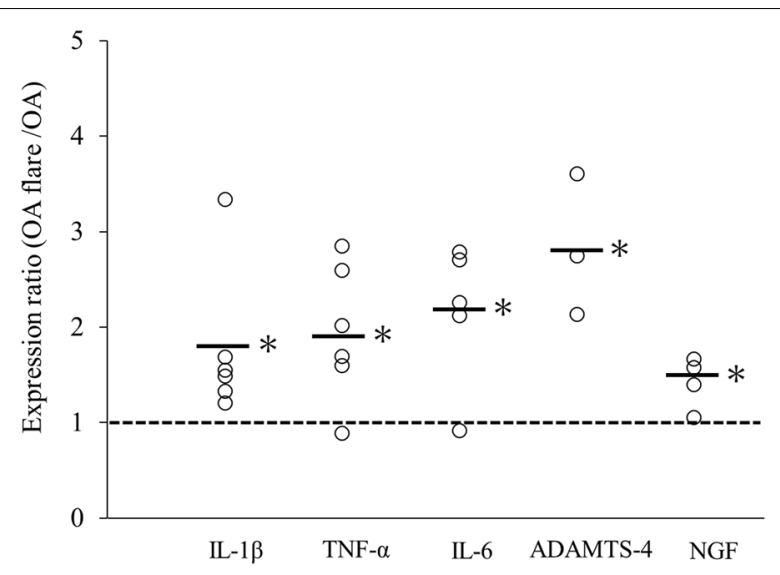

Fig. 5 Levels of mRNA expression ratio (OA flare divided by $\mathrm{OA}$ ) of IL-1 $\beta$, TNF- $a, I L-6$, ADAMTS-4, and NGF. Each plotted circle contains infrapatellar fat pad from three mice. (Bar: median *: $P<0.05$ compared with OA)

associated with disease progression compared with the no-treatment group.

\section{Role of $M C$ in osteoarthritic joint}

In OA joints, synovial MCs secrete histamine and serotonin by degranulation, which causes fibroblast proliferation and upregulates vascular permeability and neovascularization [29]. Tryptase is also released by degranulation, which is linked to cartilage matrix degradation, leukocyte recruitment, and fibroblast activation [29, 45]. Metabolites of arachidonic acid upregulate the chemotaxis of neutrophils and leukocytes, which are involved in the development of inflammation and pain. They also enhance the production of MMPs and ADAMTSs from chondrocytes, which induces further cartilage damage $[3,29]$. Proinflammatory cytokines and chemokines activate other immune cells that cause inflammation and pain [45], and induce the release of MMPs and ADAMTSs to process articular cartilage catabolism [43]. Although not all the mechanisms of action above were investigated in the current study, intraarticular injection of cultured MCs into the established OA models significantly elicited histological deterioration, pain aggravation, and elevation of IL- $1 \beta$, TNF- $\alpha$, IL- 6 , and ADAMTS-4, which partly mimics the status of acute flares and subsequent reactions in OA joints.

Recently, NGF has become particularly interesting because some clinical studies have demonstrated that injection of a monoclonal antibody against NGF provided persistent pain relief and improved function in moderate to severe OA $[18,26]$. NGF affects many painrelated molecules by signal transduction through TrkA and p75 receptors expressed on nociceptors [15, 20]. Under inflammatory conditions, NGF induces proinflammatory cytokine production from immune cells, and the synthesised cytokines further upregulate NGF expression [42]. Among the immune cells, MC plays an essential role because it can aggressively produce NGF by itself and also respond to NGF through the expression of TrkA [23]. In this study, MC injection into OA joints significantly enhanced local NGF expression and pain behavior, which possibly reinforce the rationale of this theory.

\section{MC engraftment into arthritis model}

Previously, Lee et. al reported that a mouse model that genetically lacks MCs does not cause $\mathrm{K} / \mathrm{BxN}$ serum transfer-induced ankle arthritis and histological deterioration in the joints, but after systemically engrafting MC, arthritis was observed [19]. Wang et. al showed that genetic mouse models of mast cell deficiency attenuated OA pathology induced by destabilisation of the medial meniscus, and transfusion of bone marrowderived mast cells into these mice systemically and intra-articularly reversed the relative protection conferred by MC deficiency [48]. These reports strongly support that MC plays a key role in the pathophysiology of OA. In this study, injection of exogenous MC to MC-deficient mice, which are often produced by mutation of the c-kit gene, may provide clearer results regarding the role of exogenous $\mathrm{MC}$ [11]. However, the use of MC-deficient mice in our model appears to be not necessarily suitable for estimating the role of endogenous $\mathrm{MC}$, since c-kit is expressed in various hematopoietic stem cells and bone marrow precursor cells and, consequently, mice with a mutation in the c-kit gene potentially exhibit abnormalities in the differentiation of various immune cells other than MC [28]. In this regard, our novel approach without any gene mutation may be more suitable for mimicking facilitated $M C$ infiltration in clinical situations.

\section{Relationship between exogenously engrafted $M C$ and endogenous MC in OA flare model}

Very few studies have described the fate of exogenously engrafted MCs in rodents. Nakano et al. injected MC intra-peritoneally into MC-knockout mice and confirmed viable MCs after a week [27], which was inconsistent with our immunohistochemistry results. The main reason for this discrepancy may be explained by genetic modification of animals, that is, MC-knockout or normal mice, as mentioned above. In our OA flare model, exogenous $\mathrm{MC}$ was mainly observed at first, but endogenous $\mathrm{MC}$ accounted for the majority 7 days after MC injection. This finding was unexpected but highly interesting 


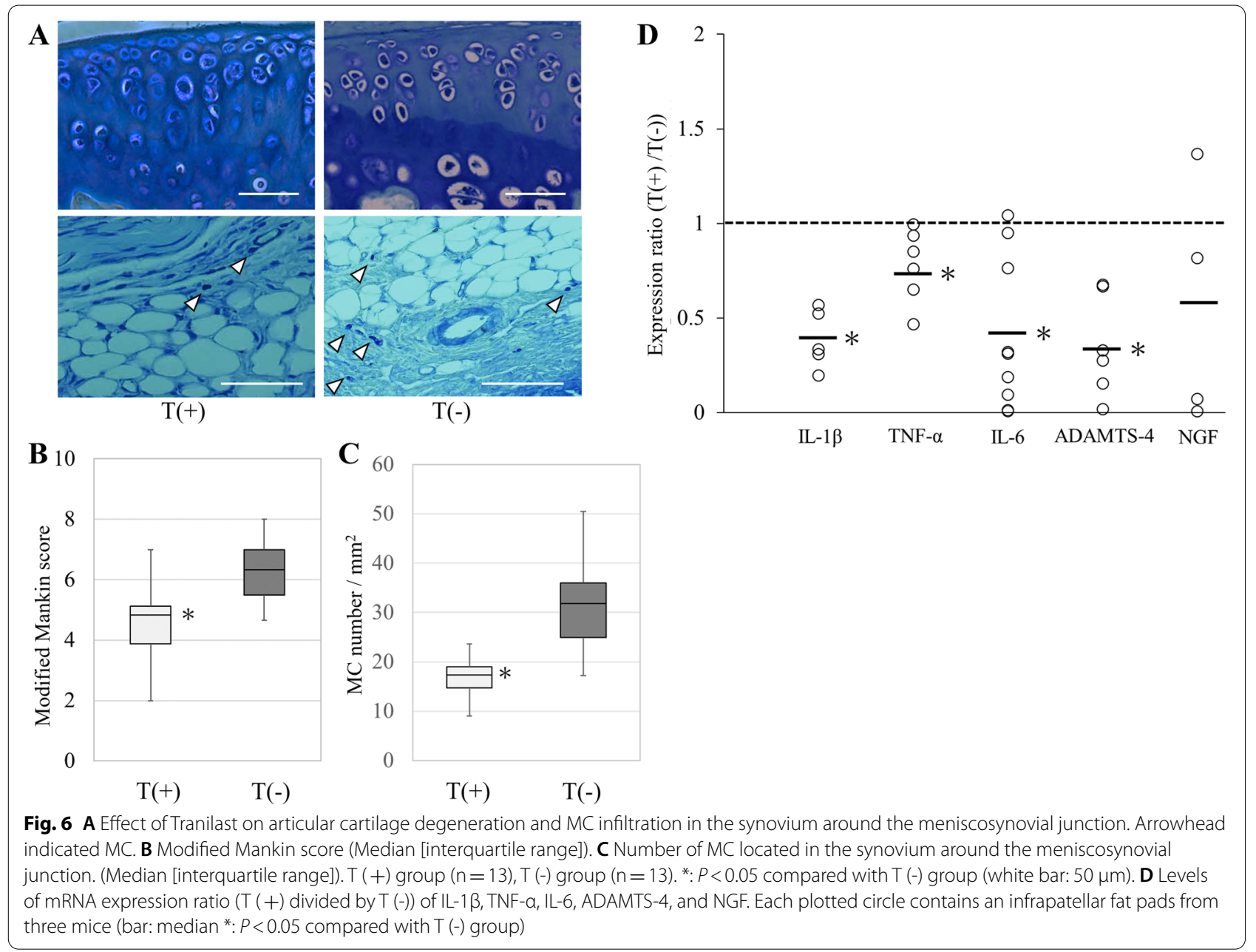

because both exogenous and endogenous MC possibly collaborated and contributed to the pathophysiology of this model. The plausible mechanism of action is as follows: Since MC injection did not work at all in the normal joints, degranulation of exogenous MC probably played a key role in OA joints and subsequently secreted proinflammatory mediators might contribute to trigger acute flares. Additionally, these mediators and various growth factors also contribute to $\mathrm{MC}$ chemotactic migration [6]. Therefore, the effect of exogenous MC would not continue for 7 days, but several mediators released from exogenous $\mathrm{MC}$ might contribute to the recruitment and activation of endogenous MC that would also work subsequently. Our OA flare model seems to have a potential advantage in investigating the molecular mechanisms through which pain is elicited by MCs in OA. However, the extent to which endogenous $\mathrm{MC}$, which is presumed to participate in the elicitation of pain in clinical status, contributed to the results could not be adequately estimated in this model.

\section{Effects of MC stabilizer}

As described, MC stabilizers seem to be a promising treatment option for acute flares in OA. Blockages of histamine, a representative molecule secreted from $M C$, was associated with a reduction in knee OA prevalence in a large observational study [41]. Tranilast is a membrane stabilizer drug that inhibits the release of various mediators from MCs. In addition to anti-allergic effects that suppress the degranulation of histamine release, tranilast inhibits the expression of many cytokines, such as IL- 6 and TNF- $\alpha[14,33,40]$, chemokines [2, 5] and proteases [38, 39]. Recent animal and human studies have also reported the efficacy and safety of MC stabilizer drugs, such as tranilast and ketotifen. Systemic administration of tranilast in a mouse collagen-induced arthritis model mimicking RA suppressed histological joint destruction and production of inflammatory cytokines and metalloproteases $[14,40]$. In a joint contracture model after trauma, ketotifen significantly decreased the number 
of myofibroblasts and $\mathrm{MC}$ in the affected joint capsule, which was associated with an improvement in contracture [24]. Furthermore, ketotifen mixed with a posttraumatic human elbow joint capsule led to decreased collagen gel contraction in vitro [13]. Consistent with these reports, our novel OA flare model demonstrated for the first time that histological OA changes, pain behavior, and gene expression of local inflammatory cytokines and aggrecanase were successfully suppressed by systemic administration of tranilast. Since the number of MCs in the synovium significantly decreased and MC-induced pain behavior was reversed in the $\mathrm{T}(+)$ group, a possible role of tranilast seemed to reduce the degranulation of the exogenous $\mathrm{MC}$, which might subsequently attenuate the activity of endogenous MC.

There are many established drugs for RA that can induce remission of the disease. However, the pathophysiology of OA remains unclear; therefore, no diseasemodifying drugs are clinically available. In this regard, tranilast is a promising candidate as a disease-modifying therapeutic drug for OA. This is because it has been used therapeutically for many years as an anti-allergic drug against a bronchial asthma, allergic rhinitis, atopic dermatitis and hypertrophic scars [44], which were all approved by the National Insurance System of our country. Of course, there are some hurdles to overcome, for example, long-lasting effects and possible adverse events in clinical trials. However, it seems to be a much easier way to reach our ultimate goal when compared to establishing new drugs.

\section{Limitations}

This study had some limitations. First, the MIA OA was a chemically induced model so that the results may not be identical to mechanically induced OA such as a meniscus resection model. Second, the contribution of other immune cells, such as macrophages and $\mathrm{T}$ cells, was not independently evaluated in this model. As the interaction of immune cells in OA pathology has received a lot of attention [7], that further studies focusing on this topic are warranted. Third, the degranulation of MCs in the OA flare model was not directly confirmed by immunohistochemistry. However, according to the literature, degranulation plays a key role in the immediate reaction of injected MC [21], which was also supported by our results of the MC non-OA model that showed no response to MC injection. Finally, the effects of $\mathrm{MC}$ injection and tranilast administration on day 22 were not investigated. Further studies are warranted to elucidate the long-term advantages of tranilast in $\mathrm{OA}$ progression and persistent pain.

\section{Conclusions}

Our novel OA flare mice model showed articular cartilage degeneration, together with $\mathrm{MC}$ infiltration at histological level, acute exacerbation of pain behavior, and increased gene expression associated with disease progression compared to the conventional MIA OA mice model. Administration of tranilast to OA flare-up provoked significant improvements in term of histological changes, pain, and gene expression. This model possibly opens a new strategy for disease-modifying treatment of $\mathrm{OA}$ focused on controlling the multiple functions of MC.

\section{Abbreviations \\ OA: Osteoarthritis; MC: Mast cell; MIA: Mono-iodoacetate; PBS: Phosphate buffered saline; BMMC: Bone marrow-derived mast cell; IL-1 $\beta$ : Interleukin-1 $\beta$; TNF-a: Tumor necrosis factor-a; IL-6: Interleukin-6; ADAMTS-4: A disintegrin and metalloproteinase with thrombospondin motifs-4; NGF: Nerve growth factor; RPMI 1640 medium: Roswell park memorial institute 1640 medium; GFP: Green fluorescent protein; MCP-6: Mast cell protease-6; mRNA: Mes- senger ribonucleic acid; RT-PCR: Real time-polymerase chain reaction; CDNA: Complementary deoxyribonucleic acid; HPRT: Hypoxanthine phosphoribo- syltransferase; RA: Rheumatoid arthritis; MMP: Matrix metalloprotease; TrkA: Tropomyosin receptor kinase A; SP: Substance P; CGRP: Calcitonin gene- related peptide.}

\section{Acknowledgements}

We would like to thank Reika Shiraishi for invaluable technical assistance.

\section{Authors' contributions}

JD was involved in planning and designing this study, the acquisition of data, analysis, and interpretation of data, and writing the manuscript. MI was involved in the conception, planning and designing this study, analysis and interpretation of data, and critical revision of the manuscript for important intellectual content. $\mathrm{HH}$ and $\mathrm{OH}$ were involved in the experiment support, acquisition of data, analysis, and interpretation of data. ST, YK, RH, and KA participated in the acquisition of data, analysis and interpretation of data. TU and MI were involved in planning this study and drafting the manuscript. All authors gave final approval of the manuscript.

\section{Funding}

This work was supported by JSPS KAKENHI Grant Number $19 \mathrm{H00372}$ and Research Grant from Nakatomi Foundation.

Availability of data and materials

The data that support the findings of this study are available from the corresponding author, $\mathrm{Ml}$, upon reasonable request.

\section{Declarations}

Ethics approval and consent to participate

All protocols in this study were approved by the institutional review board of our University for Animal research (M-00078). All methods were performed in accordance with the guidelines and regulations.

\section{Consent for publication}

Not applicable.

\section{Competing interests}

The authors have no competing interests to declare regarding the manuscript entitled: A novel mice model of acute flares in osteoarthritis elicited by intraarticular injection of cultured mast cells.

\section{Author details}

${ }^{1}$ Department of Orthopedic Surgery, Kochi Medical School, Kochi University, 185-1 Oko-cho, Nankoku, Kochi Pref, Japan. ${ }^{2}$ Multidisciplinary Pain Center, 
Aichi Medical University, Nagakute, Japan. ${ }^{3}$ Center for Innovative and Translational Medicine, Kochi University, Nankoku, Japan.

\section{Received: 2 March 2021 Accepted: 24 August 2021} Published online: 08 September 2021

\section{References}

1. Asjid R, Faisal T, Qamar K, Malik S, Umbreen F, Fatima M (2019) Effect of platelet-rich plasma on mankin scoring in chemically-induced animal model of osteoarthritis. J Coll Physicians Surg Pak 29:1067-1071

2. Baba A, Tachi M, Ejima Y, Endo Y, Toyama H, Matsubara M et al (2016) Anti-allergic drugs tranilast and ketotifen dose-dependently exert mast cell-stabilizing properties. Cell Physiol Biochem 38:15-27

3. Berenbaum F (2013) Osteoarthritis as an inflammatory disease (osteoarthritis is not osteoarthrosis!). Osteoarthr Cartil 21:16-21

4. Bridges AJ, Malone DG, Jicinsky J, Chen M, Ory P, Engber W et al (1991) Human synovial mast cell involvement in rheumatoid arthritis and osteoarthritis. Relationship to disease type, clinical activity, and antirheumatic therapy. Arthritis Rheum 34:1116-1124

5. Chikaraishi A, Hirahashi J, Takase O, Marumo T, Hishikawa K, Hayashi M et al (2001) Tranilast inhibits interleukin-1 $\beta$-induced monocyte chemoattractant protein-1 expression in rat mesangial cells. Eur J Pharmacol 427:151-158

6. Collington SJ, Williams TJ, Weller CL (2011) Mechanisms underlying the localisation of mast cells in tissues. Trends Immunol 32:478-485

7. de Lange-Brokaar BJ, loan-Facsinay A, van Osch GJ, Zuurmond AM, Schoones J, Toes RE et al (2012) Synovial inflammation, immune cells and their cytokines in osteoarthritis: a review. Osteoarthr Cartil 20:1484-1499

8. de Lange-Brokaar BJ, Kloppenburg M, Andersen SN, Dorjee AL, Yusuf E, Herb-van Toorn L et al (2016) Characterization of synovial mast cells in knee osteoarthritis: association with clinical parameters. Osteoarthr Cartil 24:664-671

9. Felson DT (2006) Osteoarthritis of the Knee. N Engl J Med 354:841-848

10. Gotis-Graham I, McNeil HP (1997) Mast cell responses in rheumatoid synovium. Association of the MCTC subset with matrix turnover and clinical progression. Arthritis Rheum 40:479-489

11. Grimbaldeston MA, Chen C-C, Piliponsky AM, Tsai M, Tam S-Y, Galli SJ (2005) Mast cell-deficient W-sash c-kit Mutant KitW-sh/W-sh mice as a model for investigating mast cell biology in Vivo. Am J Pathol 167:835-848

12. Heilborn U, Berge OG, Arborelius L, Brodin E (2007) Spontaneous nociceptive behaviour in female mice with Freund's complete adjuvant- and carrageenan-induced monoarthritis. Brain Res 1143:143-149

13. Hildebrand KA, Zhang M, Befus AD, Salo PT, Hart DA (2014) A myofibroblast-mast cell-neuropeptide axis of fibrosis in post-traumatic joint contractures: an in vitro analysis of mechanistic components. J Orthop Res 32:1290-1296

14. Inglis JJ, Criado G, Andrews M, Feldmann M, Williams RO, Selley ML (2007) The anti-allergic drug, N-(3,4'-dimethoxycinnamonyl) anthranilic acid, exhibits potent anti-inflammatory and analgesic properties in arthritis. Rheumatol (Oxf Engl) 46:1428-1432

15. Kaplan D, Hempstead B, Martin-Zanca D, Chao M, Parada L (1991) The trk proto-oncogene product: a signal transducing receptor for nerve growth factor. Science 252:554-558

16. Klein-Wieringa IR, de Lange-Brokaar BJE, Yusuf E, Andersen SN, Kwekkeboom JC, Kroon HM et al (2016) Inflammatory cells in patients with endstage knee osteoarthritis: a comparison between the synovium and the infrapatellar fat pad. J Rheumatol 43:771-778

17. Kriegova E, Manukyan G, Mikulkova Z, Gabcova G, Kudelka M, Gajdos P et al (2018) Gender-related differences observed among immune cells in synovial fluid in knee osteoarthritis. Osteoarthr Cartil 26:1247-1256

18. Lane NE, Schnitzer TJ, Birbara CA, Mokhtarani M, Shelton DL, Smith MD et al (2010) Tanezumab for the treatment of pain from osteoarthritis of the Knee. N Engl J Med 363:1521-1531

19. Lee DM (2002) Mast cells: a cellular link between autoantibodies and inflammatory. Arthritis 297:1689-1692

20. Mallett S, Barclay AN (1991) A new superfamily of cell surface proteins related to the nerve growth factor receptor. Immunol Today 12:220-223
21. Metcalfe DD, Baram D, Mekori YA (1997) Mast cells. Physiol Rev 77:1033-1079

22. Miller RE, Miller RJ, Malfait AM (2014) Osteoarthritis joint pain: the cytokine connection. Cytokine 70:185-193

23. Miller RJ, Malfait AM, Miller RE (2020) The innate immune response as a mediator of osteoarthritis pain. Osteoarthr Cartil 28:562-571

24. Monument MJ, Hart DA, Befus AD, Salo PT, Zhang M, Hildebrand KA (2010) The mast cell stabilizer ketotifen fumarate lessens contracture severity and myofibroblast hyperplasia: a study of a rabbit model of posttraumatic joint contractures. J Bone Joint Surg Am 92:1468-1477

25. Murphy SL, Lyden AK, Kratz AL, Fritz H, Williams DA, Clauw DJ et al (2015) Characterizing pain flares from the perspective of individuals with symptomatic knee osteoarthritis. Arthritis Care Res 67:1103-1111

26. Nagashima H, Suzuki M, Araki S, Yamabe T, Muto C (2011) Preliminary assessment of the safety and efficacy of tanezumab in Japanese patients with moderate to severe osteoarthritis of the knee: a randomized, double-blind, dose-escalation, placebo-controlled study. Osteoarthritis Cartilage 19:1405-1412

27. Nakano T, Sonoda T, Hayashi C, Yamatodani A, Kanayama Y, Yamamura T et al (1985) Fate of bone marrow-derived cultured mast cells after intracutaneous, intraperitoneal, and intravenous transfer into genetically mast cell-deficient W/Wv mice. Evidence that cultured mast cells can give rise to both connective tissue type and mucosal mast. J Exp Med 162:1025-1043

28. Nigrovic PA, Gray DHD, Jones T, Hallgren J, Kuo FC, Chaletzky B et al (2008) genetic inversion in mast cell-deficient wsh mice interrupts corin and manifests as hematopoietic and cardiac aberrancy. Am J Pathol 173:1693-1701

29. Nigrovic PA, Lee DM (2007) Synovial mast cells: role in acute and chronic arthritis. Immunol Rev 217:19-37

30. O'Neil CK, Hanlon JT, Marcum ZA (2012) Adverse effects of analgesics commonly used by older adults with osteoarthritis: focus on non-opioid and opioid analgesics. Am J Geriatr Pharmacother 10:331-342

31. Ogbonna AC, Clark AK, Gentry C, Hobbs C, Malcangio M (2013) Pain-like behaviour and spinal changes in the monosodium iodoacetate model of osteoarthritis in C57BI/6 mice. Eur J Pain 17:514-526

32. Ohtake-Niimi S, Kondo S, Ito T, Kakehi S, Ohta T, Habuchi H et al (2010) Mice deficient in N-acetylgalactosamine 4-sulfate 6-o-sulfotransferase are unable to synthesize chondroitin/dermatan sulfate containing $\mathrm{N}$-acetylgalactosamine 4,6-bissulfate residues and exhibit decreased protease activity in bone marrow-derived mast cells. J Biol Chem 285:20793-20805

33. Pae H-O, Jeong S-O, Koo BS, Ha H-Y, Lee K-M, Chung H-T (2008) Tranilast, an orally active anti-allergic drug, up-regulates the anti-inflammatory heme oxygenase-1 expression but down-regulates the pro-inflammatory cyclooxygenase-2 and inducible nitric oxide synthase expression in RAW264.7 macrophages. Biochem Biophys Res Commun 371:361-365

34. Pitcher T, Sousa-Valente J, Malcangio M (2016) The monoiodoacetate model of osteoarthritis pain in the mouse. J Vis Exp (111). https://doi.org/ $10.3791 / 53746$

35. Pu J, Nishida K, Inoue H, Asahara H, Ohtsuka A, Murakami T (1998) Mast cells in osteoarthritic and rheumatoid arthritic synovial tissues of the human knee. Acta Med Okayama 52:35-39

36. Ribatti D, Vacca A, Nico B, Crivellato E, Roncali L, Dammacco F (2001) The role of mast cells in tumour angiogenesis. Br J Haematol 115:514-521

37. Schneider CA, Rasband WS, Eliceiri KW (2012) NIH Image to ImageJ: 25 years of image analysis. Nat Methods 9:671-675

38. Shimizu T, Kanai K-I, Kyo Y, Suzaki H, Asano K, Hisamitsu T (2006) Effect of tranilast on matrix metalloproteinase production from neutrophils in-vitro. J Pharm Pharmacol 58:91-99

39. Shimizu T, Kanai K, Asano K, Hisamitsu T, Suzaki H (2005) Suppression of matrix metalloproteinase production in nasal fibroblasts by tranilast, an antiallergic agent, in vitro. Mediators Inflamm 2005:150-159

40. Shiota N, Kovanen PT, Eklund KK, Shibata N, Shimoura K, Niibayashi T et al (2010) The anti-allergic compound tranilast attenuates inflammation and inhibits bone destruction in collagen-induced arthritis in mice. $\mathrm{Br} \mathrm{J}$ Pharmacol 159:626-635

41. Shirinsky I, Shirinsky V (2018) H1-antihistamines are associated with lower prevalence of radiographic knee osteoarthritis: a cross-sectional analysis of the osteoarthritis initiative data. Arthritis Res Ther 20:116

42. Skaper SD (2017) Nerve growth factor: a neuroimmune crosstalk mediator for all seasons. Immunology 151:1-15 
43. Sokolove J, Lepus CM (2013) Role of inflammation in the pathogenesis of osteoarthritis: latest findings and interpretations. Ther Adv Musculoskelet Dis 5:77-94

44. Suzawa H, Kikuchi S, Ichikawa K, Koda A (1992) Inhibitory action of tranilast, an anti-allergic drug, on the release of cytokines and PGE2 from human monocytes-macrophages. Jpn J Pharmacol 60:85-90

45. Theoharides TC, Alysandratos KD, Angelidou A, Delivanis DA, Sismanopoulos N, Zhang B et al (2012) Mast cells and inflammation. Biochim Biophys Acta 1822:21-33

46. Udo M, Muneta T, Tsuji K, Ozeki N, Nakagawa Y, Ohara T et al (2016) Monoiodoacetic acid induces arthritis and synovitis in rats in a dose- and time-dependent manner: proposed model-specific scoring systems. Osteoarthr Cartil 24:1284-1291
47. van der Sluijs JA, Geesink RGT, van der Linden AJ, Bulstra SK, Kuyer R, Drukker J (1992) The reliability of the mankin score for osteoarthritis. J Orthop Res 10:58-61

48. Wang Q, Lepus CM, Raghu H, Reber LL, Tsai MM, Wong HH, et al. (2019) IgE-mediated mast cell activation promotes inflammation and cartilage destruction in osteoarthritis. eLife 8:e39905

\section{Publisher's Note}

Springer Nature remains neutral with regard to jurisdictional claims in published maps and institutional affiliations.

\section{Submit your manuscript to a SpringerOpen ${ }^{\circ}$ journal and benefit from:}

- Convenient online submission

- Rigorous peer review

- Open access: articles freely available online

- High visibility within the field

- Retaining the copyright to your article

Submit your next manuscript at $\boldsymbol{\nabla}$ springeropen.com 